\title{
Dye-based mito-thermometry and its application in thermogenesis of brown adipocytes
}

\author{
Tao-Rong Xie ${ }^{1}$, Chun-Feng Liu ${ }^{1}$, Jian-Sheng Kang ${ }^{1 凶}$ \\ ${ }^{1}$ CAS Key Laboratory of Nutrition and Metabolism, Institute for Nutritional Sciences, Shanghai Institutes for \\ Biological Sciences, Graduate School of the Chinese Academy of Sciences, Chinese Academy of Sciences, \\ Shanghai 200031, China
}

Received: 17 February 2017 / Accepted: 29 March 2017 / Published online: 13 May 2017

\begin{abstract}
Mitochondrion is the main intracellular site for thermogenesis and attractive energy expenditure targeting for obesity therapy. Here, we develop a method of mitochondrial thermometry based on Rhodamine B methyl ester, which equilibrates as a thermosensitive mixture of nonfluorescent and fluorescent resonance forms. Using this approach, we are able to demonstrate that the efficacy of norepinephrine-induced thermogenesis is low, and measure the maximum transient rate of temperature increase in brown adipocytes.
\end{abstract}

Keywords Mitochondrial thermometry, Nanothermometry, Thermogenesis, Brown adipocytes

\section{INTRODUCTION}

Temperature probing for live cells is challenging, and a lot of efforts have been made to develop nanothermometry to monitor temperatures of living cells (Ye et al. 2011; Jaque and Vetrone 2012; Li and Liu 2012; Kiyonaka et al. 2013; Kucsko et al. 2013; Arai et al. 2014, 2015; Homma et al. 2015). Recently, all such works have been challenged and criticized by Baffou et al., who have claimed no detectable temperature heterogeneities in living cells (Baffou et al. 2014). Apparently, Baffou et al. have neglected well-known facts in biology, such as the thermogenesis of brown adipocytes (BA) and mitochondrial role in thermogenesis (Cannon and Nedergaard 2004).

Tao-Rong Xie and Chun-Feng Liu have contributed equally to this work.

Electronic Supplementary Material The online version of this article (doi:10.1007/s41048-017-0039-6) contains supplementary material, which is available to authorized users.

$\bowtie$ Correspondence: jskang@gmail.com (J.-S. Kang)
Sympathetic neurotransmitter norepinephrine (NE) can mobilize free fatty acids stored in lipid droplets of BA, and dissipate electrochemical potential energy stored in mitochondrial proton gradient to product heat (Cannon and Nedergaard 2004; Fedorenko et al. 2012). Not only as the energy factory of the cells, mitochondrion is the main intracellular site for thermogenesis, which has been targeted for therapy to reduce obesity (Lowell and Spiegelman 2000; Tseng et al. 2010). Here, we demonstrate a method of mitochondrial thermometry (mito-thermometry) based on the thermosensitive characteristics of Rhodamine B methyl ester (RhB-ME). With this mito-thermometry, we revealed the low efficacy of NE-induced thermogenesis and the maximum transient rate of temperature increase in $\mathrm{BA}$, and indicated the improper critique of Baffou both practically and theoretically.

\section{RESULTS}

\section{Evaluation of RhB-ME-based mito-thermometry in HeLa cells}

Our technique for mito-thermometry employs the thermosensitive and mitochondrial targeting properties 
of RhB-ME (Fig. 1, synthesis method available online). RhB-ME (Fig. 1A and Supplementary Fig. S1A), a cationic dye like Rhodamine 800 (Rh800, a mitochondrial marker, Fig. 1B and Supplementary Fig. S1B) and many others (Sakanoue et al. 1997; Johnson et al. 1981), can redistribute within subcellular compartments in response to the negative electric potential, especially in mitochondria (Fig. 1A-D). Mitochondria with large negative membrane potentials lead to spontaneous accumulation of thermosensitive RhB-ME and thermoneutral Rh800 (Fig. 1E) in their matrixes, until reaching equilibrium in accordance with the Nernst distribution law (Sakanoue et al. 1997).

The thermochromic transformation of RhB-ME in aqueous solution results in a simple temperature profile, which can be fitted with Arrhenius equation, a single exponential model for the temperature dependence of reaction rates (Fig. 1F). The Arrhenius plot indicates that activation energy of $\mathrm{RhB}-\mathrm{ME}$ thermochromic transformation is about $-4.4 \mathrm{kcal} / \mathrm{mol}$. In living cells, to cancel out the influence of mitochondrial membrane potentials on RhB-ME concentration, the fluorescent intensity ratio of Rh800 to RhB-ME is used to represent mitochondrial thermal profile (Fig. 1G). Both RhB-ME and Rh800 are insensitive to $\mathrm{pH}, \mathrm{Ca}^{2+}$ or $\mathrm{Mg}^{2+}$ (Supplementary Fig. S2). This RhB-ME-based mito-thermometry enables us to acquire the mitochondrial thermal map of HeLa cells at room temperature (RT). The image in Fig. $1 \mathrm{G}$ shows mitochondrial temperature gradients in HeLa cells with higher temperature at the center, which can be explained by the geometry of the cells (Fig. 1C).

\section{The mechanism of RhB-ME thermochromic transformation}

RhB-ME is a methyl ester derivative of $\mathrm{RhB}$. RhB is cell membrane impermeable (Johnson et al. 1981), also thermosensitive (Supplementary Fig. S3), and exists as a mixture of nonfluorescent lactone and fluorescent zwitterion (Hinckley and Seybold 1988). Unlike RhB, our data suggest that RhB-ME exists as an equilibrium mixture of nonfluorescent mesomerism and fluorescent resonance form (Fig. 2A-C), and thermal energy $(\sim 7.5$ $k_{\mathrm{B}} T$ at $25^{\circ} \mathrm{C}$ ) can convert single RhB-ME fluorescent molecule to its nonfluorescent form (Fig. 2A). The two diethylamino groups are symmetric in the nonfluorescent form, but asymmetric in the fluorescent form (Fig. 2A). Proton nuclear magnetic resonance $\left({ }^{1} \mathrm{H}-\mathrm{NMR}\right)$ can distinguish between the symmetric form and the asymmetric form by the ${ }^{1} \mathrm{H}-\mathrm{NMR}$ spectral difference of the 12 protons from four methyl moieties in two diethylamino groups (Fig. 2B-C). The symmetric nonfluorescent form is dominant at higher temperature, which is evidenced by the undistinguishable chemical shifts of 12 protons showing single triplet in the ${ }^{1} \mathrm{H}$ NMR spectra at RT (Fig. 2B and Supplementary Fig. S4).
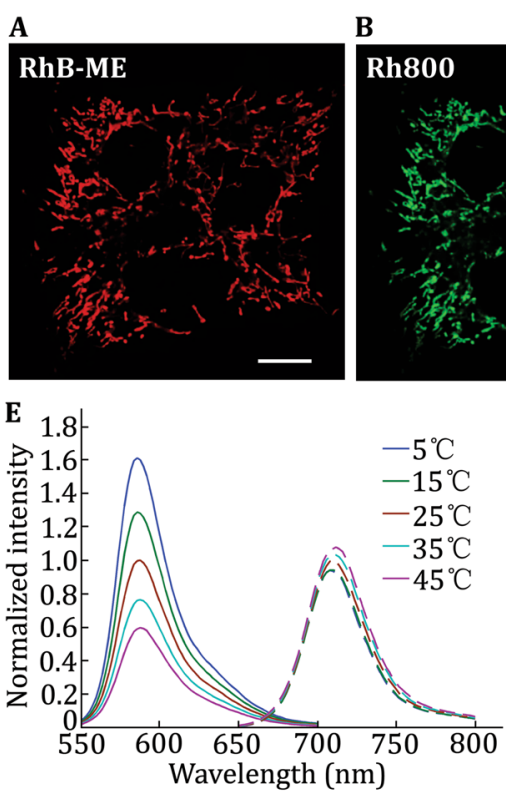

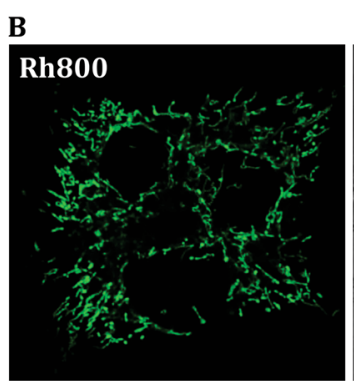

$\mathbf{F}$

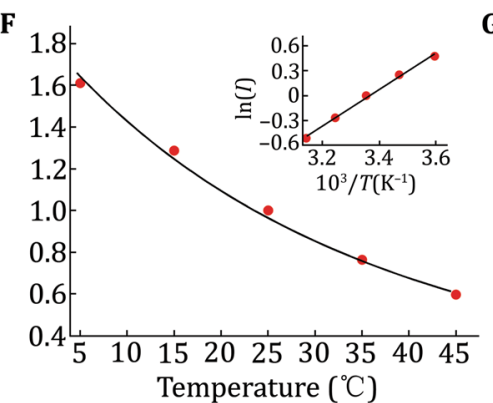

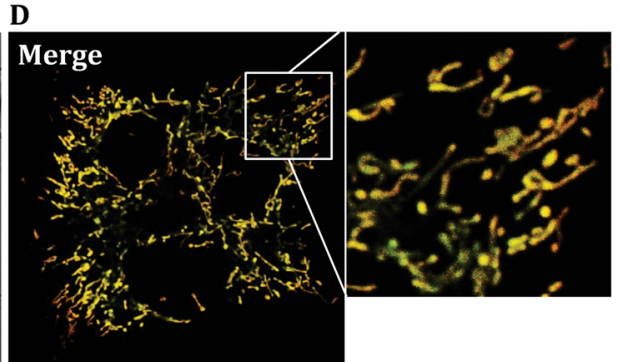

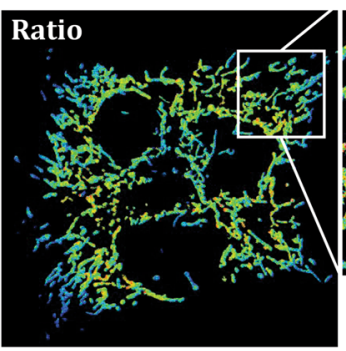

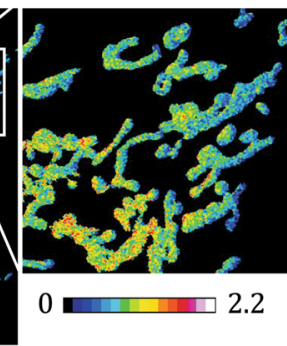

Fig. 1 RhB-ME based mito-thermometry in living cells. A-D Confocal images of RhB-ME channel (A), Rh800 channel (B), differential interference contrast (DIC) image (C) and merged image (D) of stained HeLa cells. Scale bar, $10 \mu \mathrm{m}$. E Emission spectra of $10 \mu \mathrm{mol} / \mathrm{L}$ RhB-ME (solid lines) and $10 \mu \mathrm{mol} / \mathrm{L}$ Rh800 (dashed lines) from 5 to $45{ }^{\circ} \mathrm{C}$, respectively. F Arrhenius fitting for peak values (red dots) of RhB-ME spectra. The black line indicates a fitting with Arrhenius equation. The inset shows the Arrhenius plot. G Mitochondrial thermal map of HeLa cells represented by the pseudocolor image of intensity ratio of Rh800 to RhB-ME 
A<smiles>CCN(CC)c1ccc2c(c1)Oc1cc(N(CC)CC)ccc1C2c1ccccc1C(=O)OC</smiles>

B

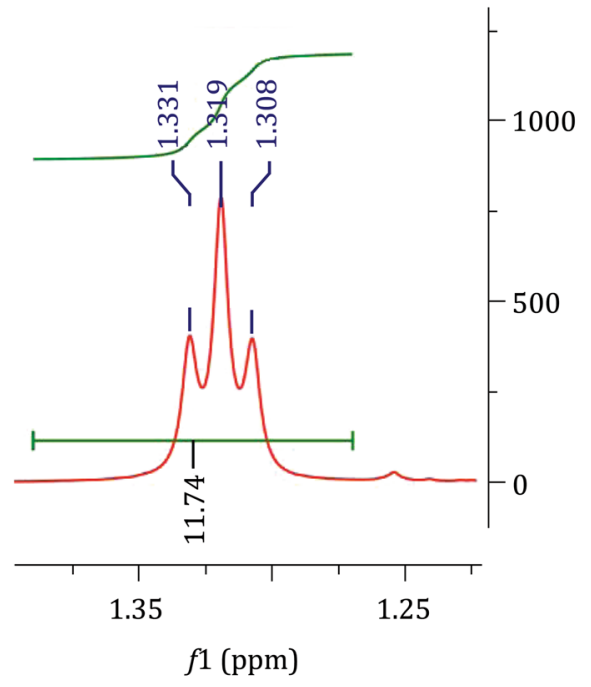

C

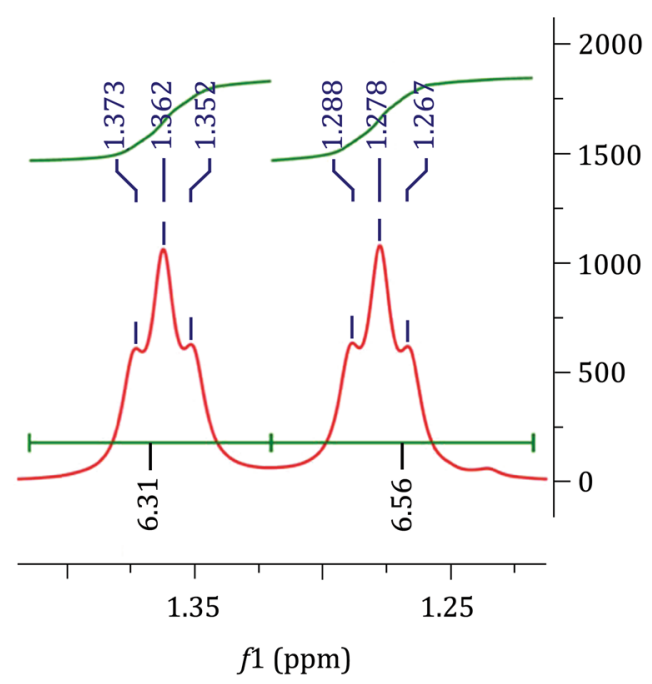

Fig. 2 The mechanism of RhB-ME thermochromic transformation. A RhB-ME exits as an equilibrium mixture of nonfluorescent and fluorescent resonance forms. B and C The ${ }^{1} \mathrm{H}-\mathrm{NMR}$ spectra of four methyl moieties in two diethylamino groups at RT (B) and $-40{ }^{\circ} \mathrm{C}(\mathbf{C})$. $\mathbf{B}$ and $\mathbf{C}$ show the chemical shifts of 12 protons in the symmetric nonfluorescent form and the asymmetrical fluorescent form, respectively

Since decreasing temperature increases the fluorescent intensity of RhB-ME (Fig. 1E, F), the asymmetric fluorescent form of RhB-ME should be dominant at low temperature, and this idea is confirmed by the chemical shifts of 12 protons splitting into double triplets in the spectral data of ${ }^{1} \mathrm{H}-\mathrm{NMR}$ at $-40{ }^{\circ} \mathrm{C}$ (Fig. 2C and Supplementary Fig. S5). Compared to thermoneutral Rhodamine 110 (Rh110, Supplementary Fig. S6) and Rh800 (Supplementary Fig. S1B), RhB (Supplementary Fig. S3) or RhB-ME (Supplementary Fig. S1A) has extra four ethyl moieties for two amino groups, which may free for torsional motion (Karstens and Kobs 1980). Thus, our findings demonstrate that thermochromic activation energy of RhB and RhB-ME is contributed to the torsional motion of diethylamino groups, which stabilizes the nonfluorescent form.

\section{Study the thermogenesis of BA with RhB-ME- based mito-thermometry}

To evaluate and make use of RhB-ME based mito-thermometry, we applied it to study the thermogenesis of BA. For thermogenic studies of BAT or BA, calorimeter and oxygen consumption rate (OCR) have been frequently used to evaluate heat production (Clark et al. 1986; Wikstrom et al. 2014), but both are indirect and might be cumbersome (Cannon and Nedergaard 2004). Although the genetically coded thermometry is versatile for organelle targeting, due to low transfection efficiency of BA, it is difficult to be used for collecting large-scale datasets, which are usually necessary for experiments with large variations. Since there is no need for transfection, injection, or elaborate 
equipment, dyes (Arai et al. 2015; Homma et al. 2015) and RhB-ME-based mito-thermometry demonstrated in this study make it easier for large-scale data acquisition, and are capable of detecting thermogenic responses at mitochondrial level.

As illustrated in Fig. 3A-D, RhB-ME, like mitochondrial marker $\mathrm{Rh} 800$, is accumulated in numerous mitochondria of primary cultured BA. The thermogenic responses of $\mathrm{BA}$, represented by the fluorescent intensity ratio of Rh800 to RhB-ME, are evoked by $0.1 \mu \mathrm{mol} /$ L NE in minutes (Fig. 3E-G, Supplementary Video S1 and S2). Compared to $10 \mu \mathrm{mol} / \mathrm{L}$ carbonyl cyanide m-chlorophenylhydrazone (CCCP, a proton uncoupling agent, Fig. 3H, I and Supplementary Video S3), $0.1 \mu \mathrm{mol} / \mathrm{L} \mathrm{NE}$ shows markedly lower thermogenic efficacy (Fig. 3J). In addition, $0.1 \mu \mathrm{mol} / \mathrm{L}$ NE-induced thermogenesis in BA shows large cell-to-cell variation (Fig. 3E, F, Supplementary Video S1 and S2). Only $59.4 \pm 15.9 \%$ (69 of 118 , mean \pm S.D.) of NE treated BA show thermogenic responses, which accounts for the low efficacy of $0.1 \mu \mathrm{mol} / \mathrm{L}$ NE-induced thermogenesis in BA (Fig. 3E, F).
A
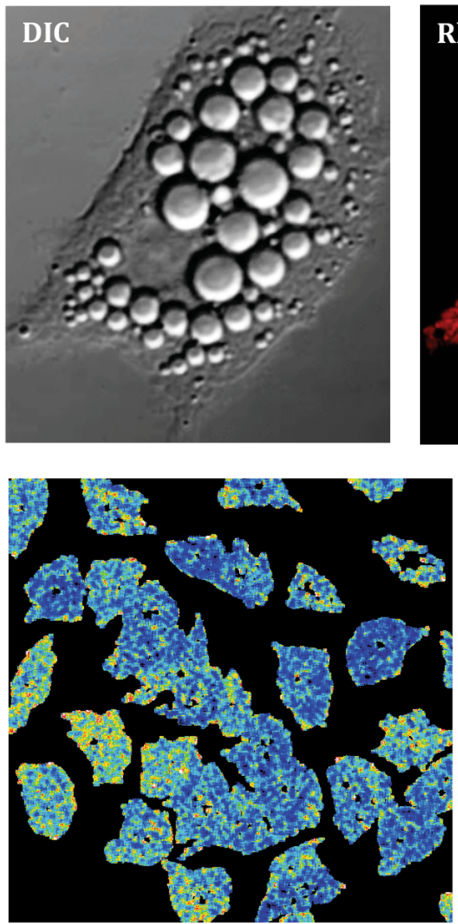

$\mathbf{H}$

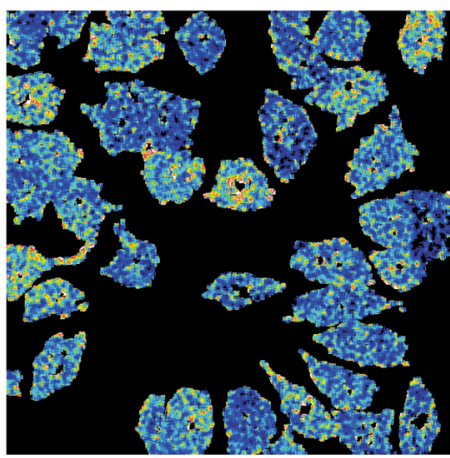

B

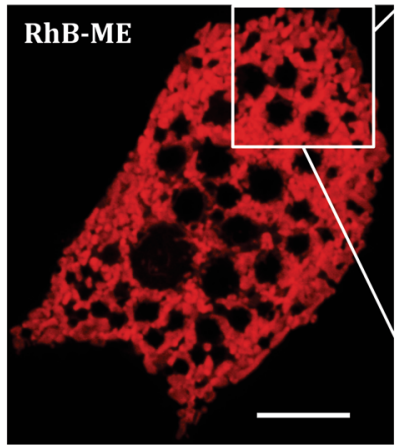

D

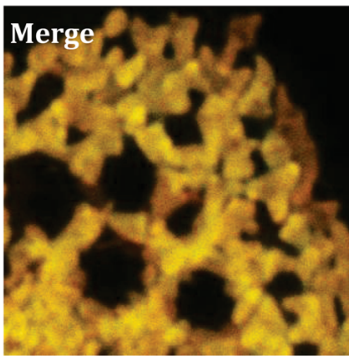

C

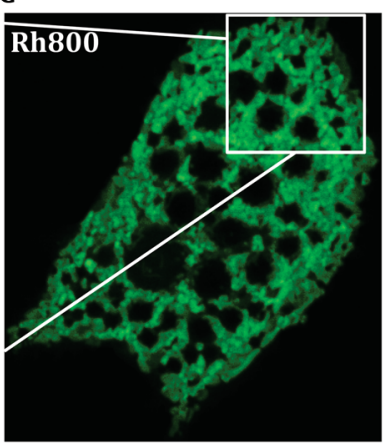

G

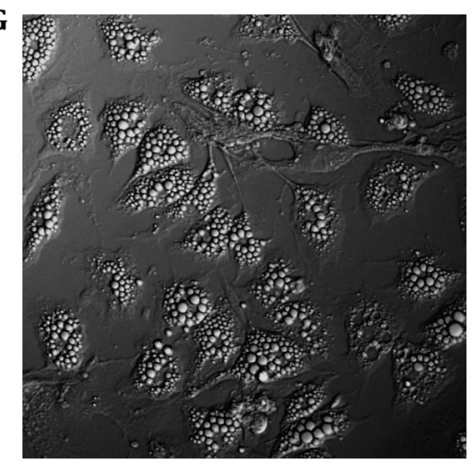

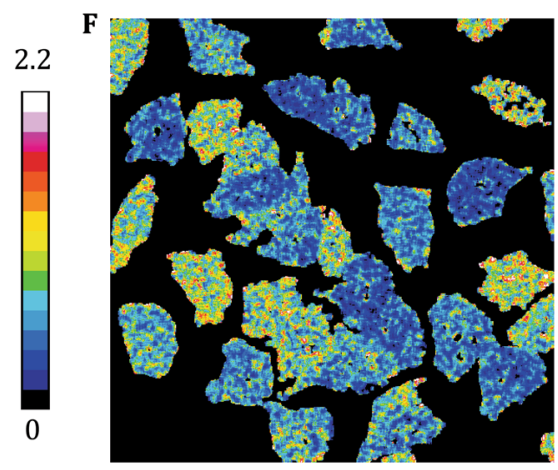
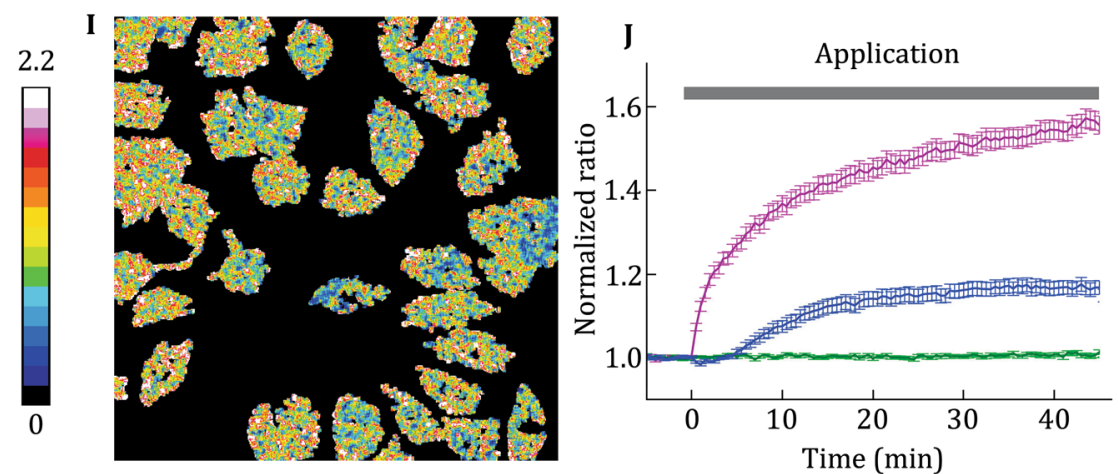

Fig. 3 Mito-thermometry reveals low efficacy of NE-induced thermogenesis in BA. A-C Confocal images of DIC image (A), RhB-ME (B), Rh800 (C) in stained BA. Scale bar, $10 \mu \mathrm{m}$. D Zoomed and merged image shows crowded mitochondria in BA. E and F Representative thermal ratio images are shown for the moments before and after NE treatment respectively. Scale bar, $20 \mu \mathrm{m}$. G DIC image of BA. H and I Representative thermal ratio images are shown for the moments before and after CCCP treatment, respectively. J NE $(0.1 \mu \mathrm{mol} / \mathrm{L}$, blue line, $n=118$ cells in four experiments $)$ and CCCP $(10 \mu \mathrm{mol} / \mathrm{L}$, magenta line, $n=88$ cells in three experiments $)$ induced thermogenesis in BA. The green line shows the control results of the solvent treatment in BA ( $n=93$ cells in three experiments). All data points in figures represent mean \pm S.E.M 

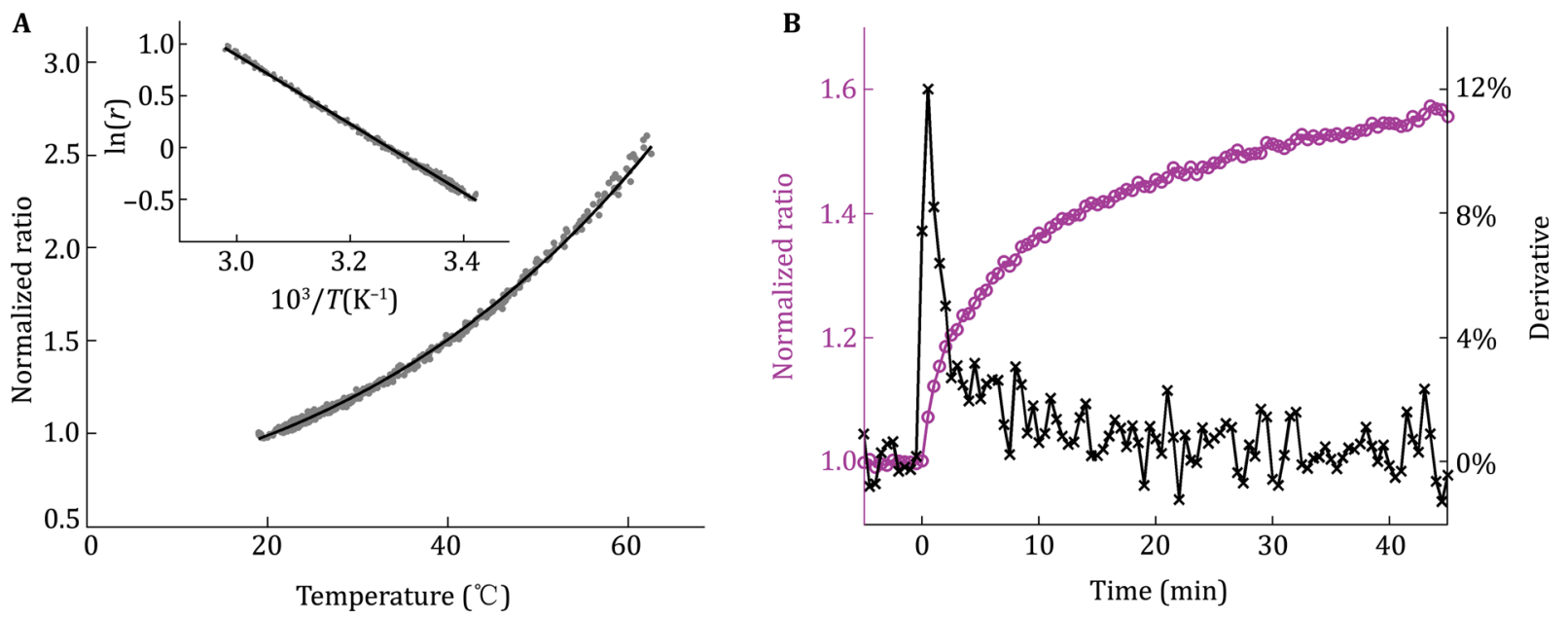

Fig. 4 Maximum transient rate of mitochondrial temperature increase in BA induced by $10 \mu \mathrm{mol} / \mathrm{L}$ CCCP. A Ratiometric confocal imaging data of $10 \mu \mathrm{mol} / \mathrm{L} \mathrm{RhB-ME}$ and $10 \mu \mathrm{mol} / \mathrm{L}$ Rh800 mixed in aqueous solution (from 18.9 to $62.9{ }^{\circ} \mathrm{C}$ ). The scatter plot of fluorescent ratios (gray dots) of Rh800 to RhB-ME versus temperature monitored with thermocouple (Pt100A). The black solid line is a fit with Arrhenius equation. The inset shows the Arrhenius plot. B Maximum transient rate of BA-mitochondrial temperature increase induced by $10 \mu \mathrm{mol} / \mathrm{L}$ CCCP. The ratio (magenta line) is the same raw dataset in Fig. 3G. The derivative (black line) of the ratio data is calculated by numerical differentiation, and the peak value is about $12 \% / \mathrm{min}$. Since all ratio data are normalized with the data acquired at $33{ }^{\circ} \mathrm{C}(306.15 \mathrm{~K})$, the change rate of normalized ratio at this temperature has been theoretically (see details in Method) and experimentally determined as $3.5 \% / \mathrm{K}(\mathrm{A})$; thus, the maximum transient rate of mitochondrial temperature increase in BA treated with $10 \mu \mathrm{mol} / \mathrm{L} \mathrm{CCCP} \mathrm{is} \sim 0.057 \mathrm{~K} / \mathrm{s}$ at $306.15 \mathrm{~K}$

\section{DISCUSSION}

Baffou et al. have criticized all methods for temperature imaging in living cells (Baffou et al. 2014), which have based on a conclusion "temperature increase should be on the order of $\Delta T \sim 10^{-5} \mathrm{~K}^{n}$ governed by the heat diffusion equation at one-dimensional steady-state conditions for cell. However, " $\Delta T$ " is a spatial temperature gradient independent of time in Baffou's model rather than "temperature increase" (over time), so it is inappropriate to apply a spatial gradient " $\Delta T$ " to discuss thermogenesis in living cells, a temporal process in time-variant systems. In addition, such small spatial temperature gradient $\left(10^{-5} \mathrm{~K}\right)$ would result in negligible heat flows within cell.

Actually, heat sources in cell would be able to increase their temperature at a transient rate, or at a lasting rate (under a condition with negligible heat dissipation) in the order of $\sim 10^{-2}-10^{-1} \mathrm{~K} / \mathrm{s}$, such as the rate $(\Delta T / \Delta t)$ of mitochondrial temperature change $(\Delta T)$, can be calculated with the Eq. 1:

$$
\Delta T / \Delta t=P / C_{\mathrm{P}, \mathrm{v}} v,
$$

which is deriving from the definition of heat capacity, and where $C_{\mathrm{P}, \mathrm{v}}$ is the volumetric heat capacity of water $\left(4.1796 \mathrm{~J} /\left(\mathrm{cm}^{3} \mathrm{~K}\right)\right) ; \quad v$ is a mitochondrial volume of $1 \mu \mathrm{m}^{3}$; the average heat power $(P)$ for a cell is $100 \mathrm{pW}$, which can reach $1 \mathrm{nW}$ for brown fat cells (Baffou et al.
2014), and then the $P$ of single mitochondrion is taken as $0.1-1 \mathrm{pW}$ assuming $\sim 1000$ mitochondria per cell.

To verify the theoretical calculation, we have estimated the maximum transient rate of mitochondrial temperature increase in $\mathrm{BA}$ induced by $10 \mu \mathrm{mol} / \mathrm{L}$ CCCP, which is indeed on the order of $\sim 10^{-2}-10^{-1} \mathrm{~K} / \mathrm{s}$ (Fig. 4). Clearly, Baffou's model is too simple to give accurate estimations since they have wrongly used the steady state without temporal factor. In addition, they have also ignored that there are various internal heat sources in cell (Ye et al. 2011; Kiyonaka et al. 2013; Kucsko et al. 2013; Arai et al. 2014, 2015; Homma et al. 2015), and that cell is nonhomogeneous and rich in membrane structures where lipid bilayer has a low thermal conductivity $(0.25 \mathrm{~W} /(\mathrm{mK})$ ) (Nakano et al. 2010).

In summary, we have practically demonstrated uneven mitochondrial thermal maps in living cells, theoretically inferred detectable heat sources (mitochondria), and also pointed out the error of Baffou's critique. RhB-ME-based mito-thermometry makes it easier for large-scale data acquisition, especially for primary cultured cells, such as BA. Our current observations raise open questions about diversely thermogenic responses of individual BA evoked by $0.1 \mu \mathrm{mol} / \mathrm{L}$ $\mathrm{NE}$, for instance, what is the in vivo regulatory mechanism to increase the efficacy of NE-induced thermogenesis in BA? 


\section{METHODS}

\section{RhB-ME synthesis}

A mixture of $\mathrm{RhB}(500 \mathrm{mg}, 1.04 \mathrm{mmol})$ and thionyl chloride $(2 \mathrm{ml})$ in chloroform $(20 \mathrm{ml})$ was heated to $60{ }^{\circ} \mathrm{C}$ and stirred for $10 \mathrm{~min}$. After cooling to room temperature, the mixture was quenched with methanol. The solvent was removed under reduced pressure and purified by prep-HPLC to give compound $375 \mathrm{mg}$, yield $73 \% .{ }^{1} \mathrm{H}-\mathrm{NMR}\left(600 \mathrm{MHz}, \mathrm{CDCl}_{3}\right) \delta 8.30(\mathrm{~d}, J=7.2 \mathrm{~Hz}$, $1 \mathrm{H}), 8.23$ (brs, 2H), 7.79-7.82 (m, 1H), 7.73-7.76 (m, $1 \mathrm{H}), 7.31(\mathrm{~d}, J=7.2 \mathrm{~Hz}, 1 \mathrm{H}), 7.06(\mathrm{~d}, J=9.6 \mathrm{~Hz}, 2 \mathrm{H})$, 6.82-6.83 (m, 4H), $3.68(\mathrm{~s}, 3 \mathrm{H}), 3.60(\mathrm{q}, J=7.2 \mathrm{~Hz}$, $8 \mathrm{H}), 1.32(\mathrm{t}, J=7.2 \mathrm{~Hz}, 12 \mathrm{H})$; ESI-HRMS exact mass calcd for $[\mathrm{M}]^{+}$requires $m / z$ 457.2486; found $m / z$ 457.2484.

\section{The change rate of normalized intensity ratio (Rh800 to RhB-ME) at a temperature}

According to Arrhenius Eq. 2, the local/pixel (j) fluorescent intensity ratio $r(j)$ of $\mathrm{Rh} 800$ to $\mathrm{RhB}-\mathrm{ME}$ at temperature $T(j)$ can be fitted with:

$$
r(j)=A \mathrm{e}^{-\frac{E_{a}}{k_{\mathrm{B}} T(j)}},
$$

where $k_{\mathrm{B}}$ is the Boltzmann constant; $E_{\mathrm{a}}$ is the observed activation energy, which is experimentally estimated $\left(E_{\mathrm{a}}=6.55 \mathrm{kcal} / \mathrm{mol}\right.$, Fig. 4$) ; A$ is a parameter related to imaging setup and experimental settings.

The parameter $A$ in Eq. 2 can be canceled out by dividing the ratio value $r_{\text {ref }}$ of a reference with a measurable temperature $T_{\text {ref. }}$ Thus, the normalized ratio $n r$ is a function of $T$ and determined by Eq. 3:

$$
n r=\frac{r}{r_{\text {ref }}}=\mathrm{e}^{-\frac{E_{\mathrm{a}}\left(\frac{1}{T}-\frac{1}{T_{\mathrm{ref}}}\right)}{k_{\mathrm{B}}} .}
$$

The change rate of $n r$ at a reference temperature $T_{\text {ref }}$ can be deduced from the derivative of Eq. 3 with respect to $T$, and determined by Eq. 4 :

$$
\frac{\Delta n r}{\Delta T}=\frac{E_{\mathrm{a}}}{k_{\mathrm{B}}} \frac{1}{T_{\text {ref }}^{2}} .
$$

Full Methods and any associated references are available in the online version of the paper.

Acknowledgements We thank the following people for their help: S.-L. You and X.-W. Liu for ${ }^{1} \mathrm{H}-\mathrm{NMR}$ and discussions; Y. Chen, H.-B. Cai, Z.-H. Sheng, D.-S. Li, Q.-W. Zhai, H. Ying and H.-X. Qi for critical reading of the manuscript; S.-L. You, X.-W. Liu, K. Hou, C. Chen and J.-J. Hao for chemical synthesis. Y.-Y. Le for the gift of HeLa cell line. This work was partially supported by National Basic Research Program of China (2011CB910903 and
2010CB912001), National Natural Science Foundation of China (31171369), Chinese Academy of Sciences (Hundred Talents Program and 20090HTP10).

\section{Compliance with Ethical Standards}

Conflict of interest Tao-Rong Xie, Chun-Feng Liu, Jian-Sheng Kang declare that they have no conflict of interest.

Human and Animal Rights and Informed Consent All institutional and national guidelines for the care and use of laboratory animals were followed.

Open Access This article is distributed under the terms of the Creative Commons Attribution 4.0 International License (http:// creativecommons.org/licenses/by/4.0/), which permits unrestricted use, distribution, and reproduction in any medium, provided you give appropriate credit to the original author(s) and the source, provide a link to the Creative Commons license, and indicate if changes were made.

\section{References}

Arai S, Lee S-C, Zhai D, Suzuki M, Chang YT (2014) A molecular fluorescent probe for targeted visualization of temperature at the endoplasmic reticulum. Sci Rep 4:6701

Arai S, Suzuki M, Park S-J, Yoo JS, Wang L, Kang N-Y, Ha H-H, Chang Y-T (2015) Mitochondria-targeted fluorescent thermometer monitors intracellular temperature gradient. Chem Commun 51:8044-8047

Baffou G, Rigneault H, Marguet D, Jullien L (2014) A critique of methods for temperature imaging in single cells. Nat Methods 11:899-901

Cannon B, Nedergaard J (2004) Brown adipose tissue: function and physiological significance. Physiol Rev 84:277-359

Clark DG, Brinkman M, Neville SD (1986) Microcalorimetric measurements of heat production in brown adipocytes from control and cafeteria-fed rats. Biochem J 235:337-342

Fedorenko A, Lishko PV, Kirichok Y (2012) Mechanism of fattyacid-dependent UCP1 uncoupling in brown fat mitochondria. Cell 151:400-413

Hinckley DA, Seybold PG (1988) A spectroscopic/thermodynamic study of the rhodamine B lactone $\rightleftharpoons$ zwitterion equilibrium. Spectrochim Acta Part A 44:1053-1059

Homma M, Takei Y, Murata A, Inoue T, Takeoka S (2015) A ratiometric fluorescent molecular probe for visualization of mitochondrial temperature in living cells. Chem Commun 51:6194-6197

Jaque D, Vetrone F (2012) Luminescence nanothermometry. Nanoscale 4:4301-4356

Johnson LV, Walsh ML, Bockus BJ, Chen LB (1981) Monitoring of relative mitochondrial membrane potential in living cells by fluorescence microscopy. J Cell Biol 88:526-535

Karstens T, Kobs K (1980) Rhodamine B and rhodamine 101 as reference substances for fluorescence quantum yield measurements. J Phys Chem 84:1871-1872

Kiyonaka S, Kajimoto T, Sakaguchi R, Shinmi D, Omatsu-Kanbe M, Matsuura H, Imamura H, Yoshizaki T, Hamachi I, Morii T, Mori Y (2013) Genetically encoded fluorescent thermosensors visualize subcellular thermoregulation in living cells. Nat Methods 10:1232-1238

Kucsko G, Maurer PC, Yao NY, Kubo M, Noh HJ, Lo PK, Park H, Lukin MD (2013) Nanometre-scale thermometry in a living cell. Nature 500:54-58 
Li K, Liu B (2012) Polymer encapsulated conjugated polymer nanoparticles for fluorescence bioimaging. J Mater Chem 22:1257-1264

Lowell BB, Spiegelman BM (2000) Towards a molecular understanding of adaptive thermogenesis. Nature 404:652-660

Nakano T, Kikugawa G, Ohara T (2010) A molecular dynamics study on heat conduction characteristics in DPPC lipid bilayer. J Chem Phys 133:154705

Sakanoue J, Ichikawa K, Nomura Y, Tamura M (1997) Rhodamine 800 as a probe of energization of cells and tissues in the nearinfrared region: a study with isolated rat liver mitochondria and hepatocytes. J Biochem (Tokyo) 121:29-37
Tseng Y-H, Cypess AM, Kahn CR (2010) Cellular bioenergetics as a target for obesity therapy. Nat Rev Drug Discov 9:465-482

Wikstrom JD, Mahdaviani K, Liesa M, Sereda SB, Si Y, Las G, Twig G, Petrovic N, Zingaretti C, Graham A, Cinti S, Corkey BE, Cannon B, Nedergaard J, Shirihai OS (2014) Hormone-induced mitochondrial fission is utilized by brown adipocytes as an amplification pathway for energy expenditure. EMBO J 33:418-436

Ye F, Wu C, Jin Y, Chan Y-H, Zhang X, Chiu DT (2011) Ratiometric Temperature Sensing with Semiconducting Polymer Dots. J Am Chem Soc 133:8146-8149 